\title{
Evaluation of Liquid Laboratory Cerebrospinal for Alzheimer's Diagnosis and Parkinson
}

\author{
Alice Prestesda Silva* \\ Anhanguera Educacional, Brazil
}

ISSN: 2637-7748

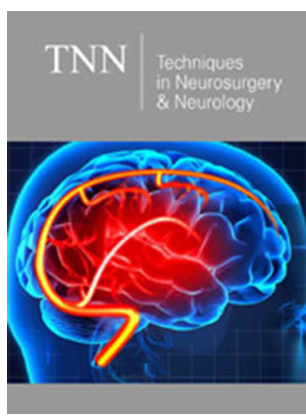

*Corresponding author: Anhanguera Educacional, Brazil

Submission: 制 December 12, 2019

Published: 毕December 01, 2020

Volume 3 - Issue 4

How to cite this article: Alice Prestesda Silva. Evaluation of Liquid Laboratory Cerebrospinal for Alzheimer's Diagnosis and Parkinson. Tech Neurosurg Neurol. 3(4). TNN. 000569. 2020.

DOI: $10.31031 /$ TNN.2020.03.000569

Copyright@ Alice Prestesda Silva, This article is distributed under the terms of the Creative Commons Attribution 4.0 International License, which permits unrestricted use and redistribution provided that the original author and source are credited.

\begin{abstract}
Alzheimer's (AD) and Parkinson's (PD) are respectivamente chronic, progressive and incurable neurodegenerative diseases. $\mathrm{AD}$ is one of the most common forms of dementia and is related to the loss of cognitive function, memory Affecting, language and behavior. PD, in turn, Mainly Affects motor coordination, tremors Causing and Difficulties in movement. The diagnosis is based on the patient's clinical history and behavioral observation, Which manifests in later stages of the disease. Magnetic resonance tomography and help in the investigation and exclusion of other pathologies. When other hypotheses are excluded, the doctor can confirm the diagnosis Then. It is called the differential diagnosis. In the search for more precious methods to investigate These diseases, diseases. Research on the socalled biomarkers CFS has shown promise and has a strong potential for clinical application in the near future, contributing to the advancement of medicine and the consequent improvement in the treatment of its carriers.
\end{abstract}

Keywords: Alzheimer's; Parkinson's; Cerebrospinal Fluid; Laboratory Diagnosis

\section{Introduction}

Alzheimer's is a neurodegenerative disease that affects mostly older people, causing a lot of impairments mainly in memory and language, in addition to Alzheimer's disease (AD), Parkinson's disease also fits under the same circumstances, the second neurodegenerative disease most common, its most obvious clinical signs are tremors. It is understood that these diseases are major concerns for public health. Many studies are being conducted on new discoveries for early diagnosis of the same, in order to offer a better quality of life for their patients, since most of the time the diagnosis is made based on clinical manifestations, which implies greater loss of neurons because symptoms may appear later such as in Parkinson's disease (PD) for example. In the search for more precious methods for investigation of these diseases, analysis of proteins presents in cerebrospinal fluid (CSF) showed to be an effective means of early identification of such diseases. The research of biomarkers in CSF has shown promise and presents a potential strong clinical application in the near future, contributing to the advancement of medicine and the improvement in the treatment of their patients. This study sought to answer the problem: "What is the purpose of the search for biomarkers for early diagnosis of Alzheimer's and Parkinson's? The overall objective of the study was to evaluate the cerebrospinal fluid of uses for the diagnosis of Alzheimer's and Parkinson's. With the specific objectives: to present as Alzheimer's and Parkinson's diseases addressing, symptoms, causes and pathophysiology; describe what they are and what are the relevant biomarkers for these diseases and compare the traditional diagnostic methods to the analysis of cerebrospinal fluid. For the development of the monograph was made and a qualitative descriptive method using as a literature review. They were studied works through Google Scholar, PubMed and SciELO, with the 15-year period. The following keywords were used: Biomarkers of Alzheimer's disease, biomarkers of Parkinson's disease, diagnosis of neurodegenerative diseases.

\section{Main Features of Alzheimer's Disease and Parkinson}

Parkinson's disease (PD) and Alzheimer's (AD) are classified as dementia which in turn, is characterized by impaired cognitive function, emotional and motivational being progressive or chronic [1]. It is estimated that in the future both developed countries, as countries that are under development will face facing the challenge of dealing with chronic diseases, which 
is dementia, predominantly in the population as a characteristic of unhealthy aging. Therefore, these facts end up affecting the quality of life of the elderly, affecting the ability of them to live in normal conditions in society and the individual, the wellbeing of the family is also impaired [2]. However, these conditions are linked to other factors such as costs for public health. The aging population creates this great financial impact. In all cases of dementia, AD is the elderly population about 60 to $70 \%$ thereof. The first description of Alzheimer's disease was just over 100 years, by Alois Alzheimer. This neurodegenerative disease is described as the most common cause of dementia, affecting around 30 million people worldwide. This pathology course is being considered an epidemic problem, with estimates that by the year 2050 the annual incidence pass to reach one million people in the United States of America.

Age is considered a risk factor, but coupled with the aging of the world population, it creates the conditions for the rapid increase in incidence. Alzheimer's is considered a neurodegenerative disease that affects mainly older people, but can occur at any age in adulthood, causing dementia. This dementia in turn, is characterized by a progressive and irreversible loss of cognitive functions, such as language and memory. It is considered a serious public health problem today. According to Fulgencio, can classify the symptoms of the disease as mild: having difficulty remembering names and also words; Moderate: memory lapses about personal events and issues, there may also be impulsive behavior; severe: alteraçõesnas skills, such as difficulties in performing daily activities, inability or communication problem. One of the important factors for the development of $\mathrm{AD}$ is the genetic associating exposure to etiological agents such as aluminum, neurotoxic amino acids, infectious agents, the occurrence of damage to microtubules and associated proteins that act directly on the genetic material, leading to somatic mutations in tissues. Patients affected with AD have a $50 \%$ chance of having children also affected by the disease. The AD brain level causes a loss of weight and volume in some areas of the brain as well as neuronal regions. The main pathophysiological characteristics of $\mathrm{AD}$ are two protein aggregates, being termed as an intracellular and extracellular senile plaque and another, and this neurofibrillary tangles. In the presence of these tangles Alzheimer proved to be fundamental for the development of the disease because its concentration and distribution over the areas of the brain are correlated with the severity of dementia. Regarding histopathology of $\mathrm{AD}$, there is a great loss of synapses, and neuronal death was observed in brain regions that are responsible for cognitive functions, being highlighted the hippocampus, cerebral cortex, entorhinal cortex and ventral striatum. These abnormalities induce the formation of neurotoxic cascade and alterations in the cytoskeleton [3-5].

First in alocorticais structures that are located in the vicinity of the hippocampus appear neurofibrillary tangles, while in the neocortex can be found amyloid plaques. In short Alzheimer's disease has characteristic pathophysiological factors, which are: microglial activation, deposition of amyloid beta peptide (Aß), herpofosforilação of Tau protein, synaptic plasticity inadequate.
From these conditions, Alzheimer's diagnosed patients have some common symptoms such as difficulty in remembering recent conversations, places, events, or names, apathy and depression, early stage considered manifestations, while the late symptoms are characterized by disorientation, impaired communication, amended trial , mood swings and personality, mental confusion, difficulty speaking, swallowing and walking. Risk factors such as, gender, genetic inheritance, depression, low education, diseases related to the disease (diabetes, dyslipidemia, obesity and hypertension) and also lifestyle collaborate significantly to the increasing incidence of Alzheimer. The survival of patients who find the disease before age 65 is 8 years and may even be least 3 years. For those who find after 90 years survival rate is 3 to 4 years. Studies predict that by the year 2030, 72 million people will be with AD. The early symptoms of AD can be quite varied, yet the disease is subdivided into early onset and late onset, but both have similar characteristics, such as the clinical neuropathology and symptoms, the difference is only in the age at which these phases are initiated in the individual. Early onset AD is also familial call, although not very common, is caused by genetic changes in some proteins, this begins before the age of 65 and their symptoms are more progressive, evolving rapidly. There is still a late-onset $\mathrm{AD}$, also known as DA sporadic, although it is common after age 65 , it is known that their disease process starts up before any clinical symptom, 2-3 decades before, specifically. In addition to Alzheimer's disease, another common neurodegenerative disease is Parkinson's disease, affecting $1.8 \%$ of adults over 65 years. This is described clinically for six cardinal motor signs: resting tremor, akinesia, rigidity, flexed posture, postural instability and gait blocks.

There are still symptoms not considered engines: dysfunction of the autonomic nervous system, cognitive and behavioral disorders, sensory and sleep disturbances. Other significant milestones of the disease are changes in handwriting, facial mimicry, mood and voice. Parkinson's disease is a multifactorial etiology that includes age, dietary habits, environmental and occupational factors as well as genetic factors. Its main feature is the degeneration of dopaminergic neurons and is characterized by a progressive and primary stage, accompanied by a mild decrease in nigrostriatal brain circuits, astrocytic glucose and the presence of Lewy bodies in the cells remaining survivors. Lewy bodies have a key constituent that is the alpha-synuclein ( $\alpha$-syn), which is a lipophilic phosphoprotein which is expressed in high amounts in neurons. It is suggested that several inflammatory cytokines are obviously increased both in blood and in the brain of the patient. These same cytokines may be similar receptor activating Toll-like receptors-TLRs. However, the causes that lead a person to develop DP are still unknown, but there are speculations of scientists that it can manifest as a result of oxidative stress generated by mitochondrial dysfunction. The prolonged use of drugs such as levodopa treatment of increased plasma homocysteine and disease are mediators that exacerbate the symptoms. Studies indicate that the characteristic motor symptoms of the disease may start decades after the cellular and molecular neuropathological symptoms, moreover, DP predominates in males aged 50 to 65 years, when 
hereditary (which may involve young adults), their symptoms may arise before age 45 . Many studies estimate that anxiety is related to the quality of life having no dependence with medical comorbidity. Moreover, in Parkinson's disease motor symptoms has great chances to get worse in the presence of anxiety symptoms. neurochemical and neuroanatomical changes are characteristic of the disease, noradrenergic and serotonergic areas of the brain stem, as well as cholinergic front regions among others, are also affected by the disease. In the midbrain are deposited multisystemic $\alpha$-synucleinopathy with Lewy bodies.

The metabolic deficiencies that may favor proteins to aggregate and premature neurodegeneration occurs. pathological conditions such as cerebral microtrauma, epigenetic changes, oxidative stress, and neuroinflammatory reactions Neuroimunes and hypoxicischemic factors are associated with Parkinson's disease. One factor that plays a central role in Parkinson's progress is oxidative stress, primarily affect the stability of the nucleic acid by oxidation of RNA, which increases the mutation of mitochondrial DNA (mtDNA) and thereby begins the synthesis of translesion (TLS) as well as disrupting the protein balance, accelerating the binding of alphasynuclein, parkin and proteasome cleavage. According to a study from 1999 to 2017 DP mortality rates among adults aged greater than 65 years increased from 41.7 to 65.3 . And the highest rates are among men in 1999 was 65.2 and in 2017 increased to 97.9. While among women it was described in 2017 43.0. It is estimated that in 2020 about 40 million people will be affected by PD with secondary motor disorders, thus requiring improvements in public health and more studies about the disease.

\section{Biomarkers of Diagnostic Relevance}

\section{What are biomarkers?}

Biomarkers also known as biological markers are present in some of our body fluids, such as saliva, blood and cefalorradiano liquid. These can be measured experimentally, indicating both normal functions such as pathological. Biological markers are divided into several types, such as physiological (organ function), histology (samples tissues obtained by biopsy), physical (changes in biological characteristics structures) and anatomical biomarkers. They can also be specific cells, genes, hormones, enzymes or molecules. However, in clinical medicine the most important for investigations are biochemical markers that are easily found in body fluids should preferably be goals, economical, reasonable, qualifying in the diagnosis and who join the condition to be searched. In the present study, the relevant biomarkers are the cerebrospinal fluid, taking into account that the molecular occurring in the brain changes reflected in the CSF, being rich source of biomarkers that could aid in the diagnosis of pathophysiological processes in the early stages mainly.

\section{Biomarkers in parkinson disease}

Studies have shown that the cerebrospinal fluid has an ideal source for bio-macardores detection of Parkinson's disease due to its proximity to the brain, they are related to increased postural instability, greater motor severity, gait changes and cognitive impairment. lower amieloide beta 1-42 levels (Aß1-42), tau protein ( $\tau$-tau) and alpha-synuclein ( $\alpha$-synuclein) in the cerebrospinal fluid were associated mainly with motor gravity; phenotype abnormal gait and postural instability. Apart from these markers, evidence has shown that serum antibodies may also be used as potential biomarkers with great specificity and accuracy for the early diagnosis of Parkinson's disease. The $\alpha$-synuclein is depicted as a cytoplasmic protein apparently deployed in their native form but depending on the environment that it is found it can be in other ways as monomers, oligomers and filamentous forms. Their primary role is not yet fully established, but it is known that it binds with the membrane and the presynaptic terminal, indicating a role in synaptic transmission. For its cytoplasmic localization, pathological implications were associated with recent studies have also shown that there may be extracellular $\alpha$-synuclein in body fluids of patients with PD. However, addition of alpha-synuclein another protein was associated with the diagnosis, this so-called DJ-1, both biomarkers in combination have a strong predictive value in detecting early disease and could identify individuals in pre-symptomatic stages, and neuroprotective treatment.

DJ-1 protein has 189 amino acids and has similarity to $\alpha$-synuclein, being expressed with amplitude in the central and peripheral nervous system, having protective role against oxidative stress, according to studies using the Western Blot technique in the cerebrospinal fluid where it was concluded that the concentration of this protein was expressed in high amounts in patients with PA compared to other controls, this result was verified in the early stage of the disease, probably due to antioxidative protective effect. By using an innovative technique called Luminex ${ }^{\circledR}$, quantification of the aforementioned proteins with increased sensitivity was allowed, which are associated with confounding variables as controls, rostro-caudal gradient CSF, blood contamination, age and sex. The conclusion was that the DJ-1 concentrations and alpha-synuclein in cerebrospinal fluid are age-dependent and are influenced by blood contamination. Mutations called "nonsense" the DJ-1 protein gene are a cause already recognized autosomal Parkinson's disease. However, the diagnosis current of PD is based around clinical symptoms, based on the incidence in populations over 65 years, with a prevalence of $3 \%$ in Brazil. To date no biomarker can be investigated through laboratory tests are available to detect early mainly of individuals likely to develop Parkinson's, this important detection to prevent their dopaminergic neurons are lost. There is speculation that brain death may result from formation of oligomers of alpha-synuclein (o- $\alpha$-synuclein) in the brain. Unfortunately, there are studies, there is evidence, but there is still availability of these biomarkers as a clinical supplement to this diagnosis [6-8].

\section{Biomarkers in alzheimer disease}

Interest in the diagnosis of early stages of Alzheimer's disease has been increasing, in turn, the priority is around the biomarkers present in the pathophysiology of the disease. The early detection 
of goal is the introduction of the drug, allowing it to be made earlier and provide better quality of life for the affected individual's interest in the diagnosis of early stages of Alzheimer's disease has been increasing, in turn, the priority is around the biomarkers present in the pathophysiology of the disease. The early detection of goal is the introduction of the drug, allowing it to be made earlier and provide better quality of life for the affected individual. Alzheimer's disease has mixed forms of dementia, being present in the cerebrospinal fluid biomarcadoes very promising for diagnosis and respective phases. As "pathological signature" three biomarkers called, are they, beta-amielóide 42 (Ab42), tau and tau-phosphorylated (phospho-tau). Changes that may be present on these biomarkers in the subject are decreased $\mathrm{Ab} 42$, which is the main constituent of the neuritic plaques; increased levels of protein tau and phospho-tau, which is the result the accumulation of intracellular neurofibrillary tangles, these originating from degeneration of neurons.

However, seeking improved diagnostic accuracy suggests is that these biomarkers are combined as an example can be mentioned that the relationship between Ab42, tau and phospho-tau can differentiate $\mathrm{AD}$ of individuals with vascular dementia. Importantly, in patients with AD CSF protein is beta-amielóide decreased, due to their arrangement in the brain parenchyma and tau protein and phospho-tau increased due to neuronal degeneration. In clinical practice examination of these biomarkers can increase accuracy in diagnosis, both in dementia phase as in mild cognitive impairment (MCI or CCL), which are intermediate between normal cognitive aging and Alzheimer's disease (dementia). These biomarkers represent correlation with the degree of neuropathological lesions, demonstrating specificity and sensitivity of around $85 \%$ to $90 \%$ for the diagnosis of AD. Moreover, the identification of the same cerebrospinal fluid can detect asymptomatic individuals at risk of developing the disease as well as pre-clinical stage of Alzheimer's, can also monitor the progression and evaluating response to treatment. Noteworthy is also the great importance of tau (p-tau) as this is related to the dysfunction of axonal transport, representing thirty-nine sites of possible phosphorylation, and its p-tau181 isoform appears with the value of $>60 \mathrm{pg} / \mathrm{mL}$ compared to healthy subjects this value is increased.

Analysis of the cerebrospinal fluid comprise complementary form in the diagnosis of various dementias. However, the evaluation of such biomarkers in the CSF should always be careful in comparison with the clinical picture, because often not all biomarkers studied have changed, fleeing the classic profile. It would be recommended that the test be done in the pre-senile stage that is before 65 years in investigative module suspected dementia. Despite the many advantages of using the present biomarkers in cerebrospinal fluid as a clinical aid of $\mathrm{AD}$, there are challenges in implementing the laboratory routine, mainly in relation to the cutting ideal values and reproducibility of the results, an example is that in several laboratories using the same biochemical analytical technique showed large variability of biomarkers levels found. There are several explanations for this to happen between them, demographic characteristics of the patients tested, disease severity, source recruitment and selection criteria and diagnosis. Other factors such as the process of getting the CSF, including collection and transportation of the sample, as well as storage conditions may interfere with the test. Also, interference is included like the choice of the test kits used for these variations may have, laboratory equipment and variability of the test procedure. Finally, it is extremely important that each laboratory has established its own reference values and a reproducible methodology used in other laboratories so that tests be included in routine laboratory and contribute significantly to the early diagnosis of Alzheimer's disease.

\section{Methods for Alzheimer's Diagnosis and Parkinson}

\section{Traditional diagnosis for Alzheimer}

As an initial assessment for Alzheimer's diagnosis is counted with neuroimaging tests such as computed tomography (CT) and magnetic resonance imaging (MRI). The tomography can be used to differentiate reverse and secondary causes such as subdural hematomas, normal pressure hydrocephalus and tumors. However, MRI to be able to greater anatomical details of the brain and change detection is the method of choice, except when the patient cannot take the exam because there are contraindications. In relation to anatomical aspects of Alzheimer there hippocampal atrophy dependent on a skilled viewer, this aspect has a specificity of 80 to $85 \%$ in differentiating $\mathrm{AD}$ from normal individuals, but in that respect the sensitivity that is lower for patients who have impaired mild cognitive. It is also possible to perform tomography emission tomography (PET), which allows visualization of the deposition of beta-amielóide. It is evident that the reduction of cerebral blood flow may be identified by computed tomography single photon emission tomography (SPECT) is also a hallmark of Alzheimer's disease, but not specific. As previously mentioned with the aid of PET can visualize and quantify the $\beta$-amyloid protein, but cannot bind to the amorphous and diffuse aggregates, which are the diffuse plaques, neurofibrillary even the braids. It is important to note that the diagnosis of AD cannot be performed if the patient's consciousness is affected by, delirium, drowsiness, other clinical abnormalities, coma, or anything that may harm the correct evaluation of the mental state [9-11].

\section{Traditional diagnosis for Parkinson}

It is known that Parkinson's disease is marked by symptoms of both engines, as no engines, and these appear until decades before the classic symptoms of the disease, tremors. The diagnosis of PD, in turn, is made based only on the symptomatology clinical symptoms engines, thus detected in late stages of the disease, namely disease is diagnosed only when about $80 \%$ of striatal dopamine and $60 \%$ of the cells dopaminergic substantia nigra have already been destroyed. The diagnosis is made in full confidence to the patient, being considered from two to four cardinal symptoms such as rigidity, resting tremor, bradykinesia and postural instability. It can 
still be considered as a complement to research the response to levodopa. The significance of the observation is significant tremor at rest, being present in $85 \%$ of patients with Parkinson's, which comes down to an extremely complicated diagnosis in the absence of the symptom. The diagnosis is accurate only with an autopsy, which can be confirmed.

\section{Cerebrospinal fluid analysis}

The cerebrospinal fluid (CSF) also known as CSF is a biological fluid binds closely with the central nervous system. Being a ultrafiltrate produced by the choroid plexus, is the cerebral ventricles and the subarachnoid space. Therefore, tests from the same are of great importance for evaluations, including diagnosis and monitoring of neurological diseases. Yet still needs further studies around the stability of the analytes during the preclinical phase. Its main function is to mechanically protect the brain, i.e., serving as a "shock absorber" and the spinal cord plays the role of protection against shock and pressure. Still has the buoyancy of defense against infectious agents that can attack the central nervous system, removes waste and facilitates the circulation of nutrients, maintaining the dynamism of the elements.

Regarding its analysis, the liquor to be considered normal protein should consist of concentrations of glucose, lactate, enzymes, potassium, magnesium and somewhat elevated concentrations of sodium chloride. In their composition should be as five cells per cubic millimeter, having an aspect called "rock water", which turn colorless and clear. However, pathological conditions, the cerebrospinal fluid may get cloudy, justifying the increase in cells ( $>400$ cells $\left./ \mathrm{mm}^{3}\right)$, but also for growth of bacteria or fungi. The classification of color, it can be classified as xanthochromic or eritrocrômico respectively mean that the yellowing caused by the presence of bilirubin, which can result from a subarachnoid hemorrhage or protein transudation phenomenon and a reddish color due to hemolysis of erythrocytes can be caused by bleeding that can result from a puncture accident for instance, or subarachnoid hemorrhage. The protein analysis in the CSF should provide from 0 to $40 \mathrm{mg} / \mathrm{dl}$ in the lumbar level, $80 \%$ of the proteins are found from the plasma and the $20 \%$ are produced in the central nervous system. The protein concentration is increased protein level in CSF is due to changes of the blood-CSF barrier or intralecal immunoglobulin synthesis. Changes to the protein may still have to do with neurological disorders such as cerebral edema, meningitis, tumors of the central nervous system, metabolic disorders, leukemia, among others. Cerebrospinal fluid is also of great importance consisting of biomarkers such as tau protein, peptide A $\beta 1-42$ (beta-amielóide).

\section{Important implementation biomarkers gifts in liquid cerebrospinal}

The main objective of biomarker discovery for Parkinson's disease, is its sensitivity for the detection of individuals who do not yet have the motor symptoms, which are in the preclinical phase and its distinction from other conditions that may present similar symptomatology with SD. The ability to diagnose early gives the patient a chance to more effective treatment as yet there is no cure. The urgent need to develop reliable and economical biomarkers relates also with the aim of early intervention, so that you can monitor therapeutic methods that could delay or stop the course of the disease, so can contribute to reducing the inability of the person affected by Parkinson. Regarding the complementary diagnosis of Alzheimer's disease, to identify biological markers in cerebrospinal fluid indicates detection accuracy both in phase and in dementia mild cognitive impairment and specifically AD.

Further, more biomarkers correlate with neuropathologic lesions, demonstrating sensitivity and specificity of approximately 85 to $90 \%$ for the diagnosis of disease. The biomarkers can be applied to the identification of asymptomatic individuals, but that present the risk of developing pre-clinical stage of Alzheimer's, can monitor the progression of the disease and to evaluate their response to treatment. A major challenge is to distinguish between cognitive changes of normal aging are specific manifestations of the early stages of dementia disorders, particularly Alzheimer's disease. Individuals who carry mild cognitive impairment has great chances to progress to dementia in later years, getting high on the pre-clinical manifestation of AD. Therefore, justified the search of biomarkers that can be done early diagnosis of Alzheimer's disease. A study where it was found that mild cognitive impairment patients showed changes in CSF biomarkers, completing a risk 17-fold higher than individuals that do not show changes in the proteins used as biomarkers. The combination of the three biomarkers (beta-amielóide, tau and phosphorylated tau) were sensitive to investigate the evolution of Alzheimer's disease, moreover the correlation thereof with the structural evaluation of the central nervous system (CNS), particularly the increase the hippocampus, is predicted better insulated ratings [11-15].

\section{Result}

Parkinson's and Alzheimer's diseases, disorders studies are lacking about its diagnostic laboratory using the cerebrospinal fluid (CSF) as a search source. It is expected that in the future there will be more concrete materials from this examination, since it is very important, especially for early diagnosis. With the evolution of CSF assessment results for their illnesses, it is estimated that treatment is more effective when the diagnosis is not Late, as AD and PD are manifested up to ten years before symptoms present in the individual. Finally, with a more accurate diagnosis, as well as providing an improvement in the patient's quality of life, allows more discoveries about the etiology of diseases, as some proteins are not fully proven that are present in them. Therefore, the more studies, more medical aid has.

\section{References}

1. Cacabelos R (2017) Parkinson's disease: from pathogenesis to pharmacogenomics. Int J Mol Sci 18(3): 551.

2. Carmelli (2011) Alzheimer's disease diagnosis in Brazil. Dement Neuropsychol 5(1): 11-20.

3. COSTA (2011) Biomarkers in cerebrospinal fluid for the diagnosis of early Parkinson's disease. Portugal. 
4. COSTA (2013) Isabela parisi. Neurobiology of Alzheimer's disease. Biosciences Institute of Rio Claro, Brazil.

5. Maria, Emilene R, Silvana SK, André LP (2018) Diagnostic validity of biomarkers in Parkinson's Disease: systematic review and metaanalysis. Rev Bras Enferm 71(6): 3074-3083.

6. Dantas, Anny Motta C, Frank, Andrea A, Abreu E (2008) Antioxidant vitamins in Parkinson's disease. Rev bras Geriatr gerontol Rio de Janeiro 11(1): 105-116.

7. Dimas, Luciana F, Sohler, Marzia P (2008) Examination of cerebrospinal fluid: influence of temperature, time and analytical sample preparation in stable. Bras Pathol Lab Med 44(2): 97-106.

8. Diniz, Breno SO, Orestes V (2007) Ousode biomarkers in the cerebrospinal fluid in the diagnosis of early Alzheimer's disease. Rev Psiquiatr Clín São Paulo 34(3): 144-145.

9. Falco, Anna (2015) Alzheimer's disease: etiological hypotheses and perspectives of treatment. Quim New, pp. 1-18.
10. Stephanie Martins (2019) Impact of anxiety symptoms on quality of life in Parkinson's disease: a systematic review. J Bras Psiquiatr Riode Janeiro 68(1): 48-55.

11. Goldman, Posthumous, R (2014) Premotor and nonmotor features of Parkinson's disease. Current Opinion in Neurology 27(4): 434-441.

12. Carolina AM (2015) Biomarkers of cerebrospinal fluid for the differential diagnosis of Alzheimer's disease. J Bras Pathol Med Lab Riode Janeiro 51(6): 376-382.

13. Moreira, Camilla Silveira (2007) Parkinson's disease: how to diagnose and treat. Scientific Journal of the Faculty of Medicine Field 2: 2.

14. Sereniki, Adriana (2008) Alzheimer's disease: physiological and pharmacological aspects. Rev Psiquiatr Rio Gd Sul Porto Alegre 30(1): $1-12$.

15. Steidl, Eduardo (2007) Parkinson's disease: literature review. Disc Science Series Health Sciences Santa Maria 8(1): 115-129. 